\title{
Combining Polynomial Chaos Expansions and Genetic Algorithm for the Coupling of Electrophysiological Models ${ }^{\star}$
}

\author{
Gustavo Montes Novaes ${ }^{1,3[0000-0002-1484-5093]}$, Joventino Oliveira \\ Campos $^{1,3[0000-0003-1899-9441]}$, Enrique Alvarez-Lacalle, ${ }^{2}$ [0000-0001-6824-6857], \\ Sergio Alonso Muñoz ${ }^{2[0000-0002-3989-8757]}$, Bernardo Martins \\ Rocha ${ }^{1[0000-0001-8978-4718]}$, and Rodrigo Weber dos \\ Santos ${ }^{1[0000-0002-0633-1391]}$ \\ 1 Post-graduated program in Computational Modeling, Federal University of Juiz de \\ Fora, Juiz de Fora MG, Brazil \\ 2 Department of Physics, Universitat Politècnica de Catalunya-BarcelonaTech, 08028 \\ Barcelona, Spain \\ 3 Department of Computation and Mechanics, Federal Center of Technological \\ Education of Minas Gerais, Leopoldina MG, Brazil \\ gtvmontes, joventinoo@gmail.com, enric.alvarez, s.alonso@upc.edu, \\ bernardo.rocha, rodrigo.weber@ufjf.edu.br
}

\begin{abstract}
The number of computational models in cardiac research has grown over the last decades. Every year new models with different assumptions appear in the literature dealing with differences in interspecies cardiac properties. Generally, these new models update the physiological knowledge using new equations which reflect better the molecular basis of process. New equations require the fitting of parameters to previously known experimental data or even, in some cases, simulated data. This work studies and proposes a new method of parameter adjustment based on Polynomial Chaos and Genetic Algorithm to find the best values for the parameters upon changes in the formulation of ionic channels. It minimizes the search space and the computational cost combining it with a Sensitivity Analysis. We use the analysis of different models of L-type calcium channels to see that by reducing the number of parameters, the quality of the Genetic Algorithm dramatically improves. In addition, we test whether the use of the Polynomial Chaos Expansions improves the process of the Genetic Algorithm search. We find that it reduces the Genetic Algorithm execution in an order of $10^{3}$ times in the case studied here, maintaining the quality of the results. We conclude that polyno-
\end{abstract}

\footnotetext{
* Supported by organizations Coordenação de Aperfeiçoamento de Pessoas de Nível Superior (CAPES), Fundação de Amparo à Pesquisa do Estado de Minas Gerais (FAPEMIG), Conselho Nacional de Desenvolvimento Científico e Tecnológico (CNPq), Universidade Federal de Juiz de Fora (UFJF), Centro Federal de Educação Tecnológica de Minas Gerais (CEFET-MG) and E. Alvarez-Lacalle acknowledges funding from Spanish Ministry for Science, Innovation and Universities under grant SAF2017-88019-C3-2-R.
} 
mial chaos expansions can improve and reduce the cost of parameter adjustment in the development of new models.

Keywords: Dimensional Reduction · Emulation · Genetic Algorithm.

\section{Introduction}

The first mathematical models that simulate the electrical activity of heart cells use Hodgkin-Huxley [9] type equations to describe the ions channels present in the cell membrane $[2,20]$. This type of equations is well consolidated in whole-cell models since it is the cornerstone of full-heart models which tries to reproduce organ-scale behavior.

However, these models fail when cardiac cells do not present homogeneous properties, such as differences in calcium concentrations due to intracellular calcium waves, across the cell. Indeed, recent intracellular models have introduced stochastic equations based on Markov Chains [13]. These Markov Chains formulations reflect the structure of the ion channels and are critical to any intracellular model. However, precisely because of its better physiological relation with reality, this new type of formulation has also found its way as average non-stochastic equations useful for homogeneous whole-cell models [16]. For instance, studies involving different mathematical models of cardiomyocytes have shown that the $I_{C a L}$ current is better modeled when it is formulated using Markov Chains since it may reproduce the different states that a single channel can assume $[1,3]$.

The use of the older models still remain useful but some studies $[7,18]$ show an effort to update the old formulations in order to reproduce new experiments and improve the biological meaning of the equations. In this update process, it is common to merge different models due to the fact that each one might complement the other. A very common issue that arises from this coupling process is that they may generate inconsistent results since each model has different assumptions in its conception. To correct these problems a parameter adjustment has been an effective tool. However, the way how this process is done has not been studied properly and presents a wide range of different scenarios.

One of the analyses that have been used in recent studies $[8,10,11]$ to see how the parameters are associated with the biological behavior is Uncertainty Quantification (UQ). Since this analysis considers the existence of uncertain measures associated with the studied object, it provides some methods to quantify the impacts of uncertainty in these parameters values upon model outputs. A technique used to perform this analysis is the polynomial chaos expansion (PCE), which approximate model outputs through orthogonal polynomials in terms of the uncertain model inputs[19].

Therefore, the main objective of this paper is to study and propose a systematic process to merge different ionic models involving parameter adjustment based on polynomial chaos expansion and genetic algorithm. 


\section{Methods}

\subsection{Modeling the L-type Calcium Current. The Pandit-Mahajan Baseline Model}

We take the rat ventricular electrophysiological model developed by Pandit et $a l .[14]$ as the structural model we are going to use. The original Pandit formulations models calcium, sodium and potassium channels to reproduce the action potential of the rat's ventricle. The calcium current entering the cell via thousand of L-type calcium channels $I_{C a L}$ is written in terms of the Equation (1).

$$
I_{C a L}=g_{C a L} d\left[\left(0.9+\frac{C a_{\text {inact }}}{10.0}\right) f_{11}+\left(0.1-\frac{C a_{\text {inact }}}{10.0}\right) f_{12}\right]\left(V-E_{C a L}\right)
$$

where the parameter $g_{C a L}$ is the maximum conductivity, $d$ is associated with gating activation, $f_{11}, f_{12}$ and $C a_{\text {inact }}$ are parameters associated with the gating inactivation, $V$ is the transmembrane potential and $E_{C a L}$ is the Nernst potential associated with the $C \mathrm{Ca}^{2+}$ ions ${ }^{1}$.

The above equation fits the experimental average calcium entering into the cell due to thousands of L-type calcium channels. It does not take into account, however, the typical structure of the L-type calcium channel. We take as a typical benchmark of any model development the ability to replace the Pandit formulation based on Hodgkin-Huxley approach with a more detailed averaged formulation of Markovian states. We take the formulation for the L-type $\mathrm{Ca}^{2+}$ as a Markov Chain developed by Mahajan et al. for rabbit which reads

$$
I_{C a L}=g_{C a L} O_{M}\left(V-E_{C a L}\right)
$$

where $O_{M}$ is the state of the Mahajan Markov Chain that is associated with the channel open state. The model of the LCC has two relevant closed states and two inactivated, besides a single open state. As can be seen, the new formulation represents a single replacement in the usage of the gating formulations to the Markov Chain formulations ${ }^{2}$.

Given that the original models were developed to simulate the electrophysiology of different animals, a parameter adjustment is necessary to make the two parts of the coupled model compatible. Figure 1 shows the difference between the original Pandit $I_{C a L}$ and Action Potential (AP) curves and the same curves where the Mahajan model of LCC has been introduced without any parameter adjustment (we will call this model the Pandit-Mahajan baseline). As can be seen in Figure 1, the new $I_{C a L}$ current was too small with respect to the Pandit original values. Thereby, this difference ends up influencing the main model values, as shown by the AP curves.

\footnotetext{
${ }^{1}$ For more details about the original Pandit $I_{C a L}$ equation, see Pandit et al. [14].

2 For more details about the transition rates between LCC rates and its dependence with voltage and calcium, see Mahajan et al.[13].
} 


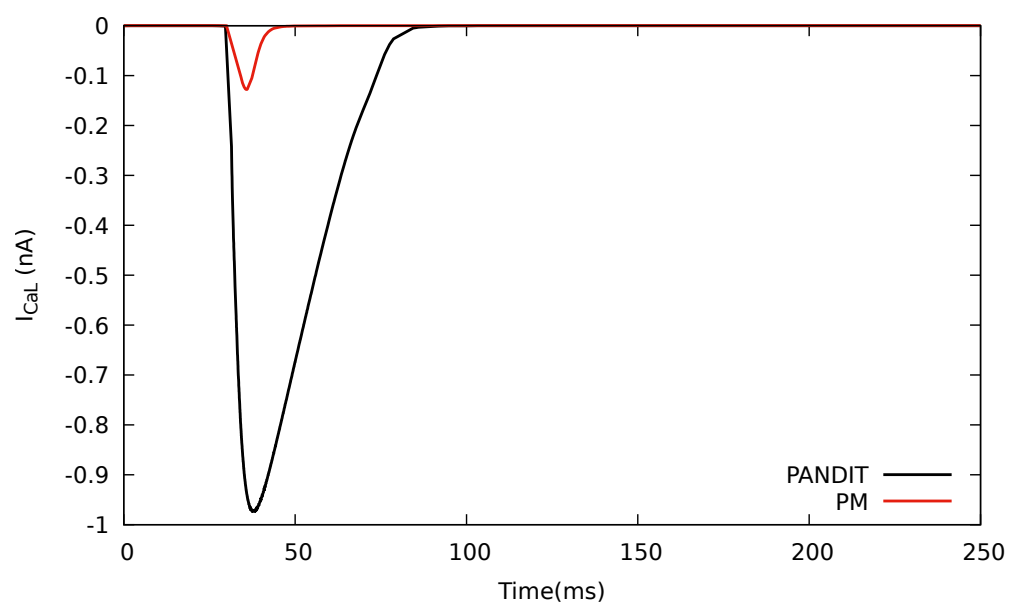

(a)

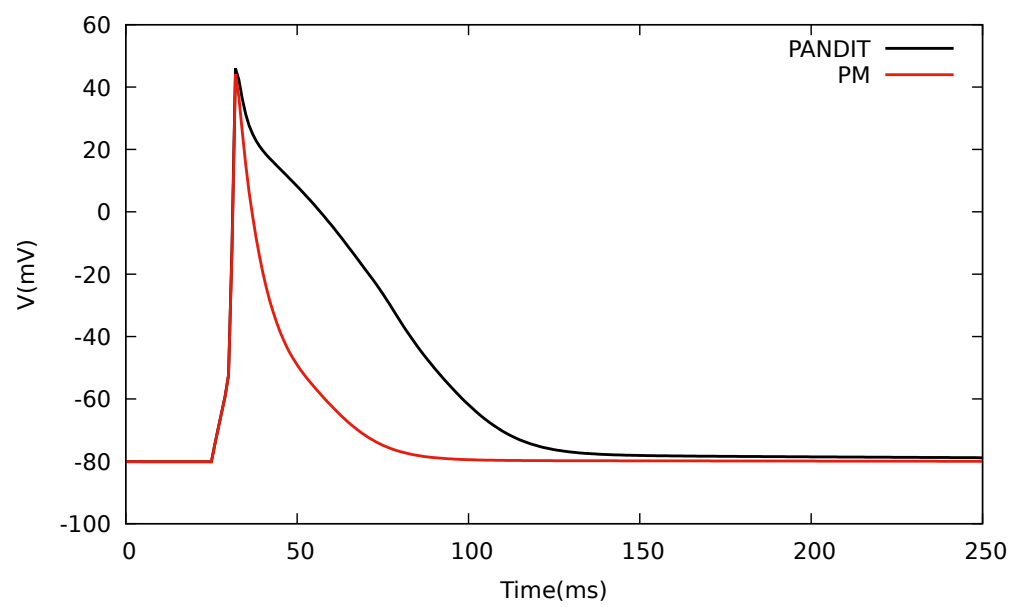

(b)

Fig. 1. Comparison of the $I_{C a L}$ (a) and AP (b) curves of the original Pandit model (PANDIT) with the $I_{C a L}$ and AP curves generated after Pandit-Mahajan (PM) coupling.

In order to describe the $I_{C a L}$ curve we will use the "Time to Peak", which is the time that the current take from the beginning of the channels opening up to the moment when it reaches the minimum value; "Peak Value", which is the minimum value that the current reaches; and "Time to Decay", which is the time that the current take from the minimum value up to the channels close. The Pandit model has values of "Time to Peak", "Peak Value" and "Time to Decay" of $6.86 \mathrm{~ms},-0.97 \mathrm{nA}$ and $38.77 \mathrm{~ms}$, respectively. While Pandit-Mahajan 
baseline model (without any adjustment) has $3.04 \mathrm{~ms},-0.13 \mathrm{nA}$ and $3.55 \mathrm{~ms}$. So, analyzing these features of the two model, Pandit and the baseline PanditMahajan, it is possible to conclude that a parameter adjustment is necessary.

It is clear now the aim of our work: we want to obtain a reliable PanditMahajan model which reproduces the results of the rat ventricle (Pandit) using the formulation of the LCC obtained in the study of the physiology of the rabbit.

The LCC Markov Chain from Mahajan has a total of seven states and twenty transition rates. However, a considerable number of these rates is a combination of others. So, in the parameter fitting process, those composed rates was not considered. After that, eight transition rates $-k_{1}, k_{2}, k_{1}^{\prime}, k_{2}^{\prime}, r_{1}, r_{2}, s_{1}$ and $s_{1}^{\prime}$ - and the parameter $\tau_{p o}$ (time constant of activation associated with the $\alpha$ and $\beta$ transition rates) together with the Pandit's maximum conductance of $I_{C a L}$ current - $g_{C a L}$ - were considered in this study.

The parameter adjustment process may be done using a Genetic Algorithm. However, a minimization problem solved by a genetic algorithm may become computationally expensive depending on the dimension of the search space. In the case of parameter adjustment from the baseline Pandit-Mahajan model, the GA would have to find a set of ten values for the parameters so that those values should reproduce the original Pandit $I_{C a L}$ current values. In this search space, it might be difficult to find the optimal values.

To reduce the GA search space, Sensitivity Analysis and Dimensional Reduction techniques were used to determinate how the parameters of the coupled model influence the main $I_{C a L}$ characteristics. With this analysis, it is possible to choose the most relevant parameters in order to adjust the new $I_{C a L}$ current. Furthermore, the search process has to simulate the mathematical model several times and, due to it, the computational cost associated with this process becomes expensive. Trying to minimize it, the use of Emulations [10,11] based on Polynomial Chaos Expansion was tested in order to replace the mathematical model in the evaluation process of the GA.

\subsection{Polynomial Chaos Expansion (PCE) and Emulations}

Emulations, also known as Metamodels in the literature, has a very important role in the study involving complex systems models [15]. This technique aims to decrease the complexity of the mathematical models caused by the overparametrization but maintaining the results as expected. With this process, the new model loses a few phenomenological information but, on a high scale, it is still able to reproduce the expected results and to help on the decision-making process.

In studies involving the Uncertainty Quantification, a common technique to analyze the insertion of uncertainty in deterministic models is the Polynomial Chaos Expansion (PCE). In this technique, an orthogonal polynomial is used to approximate some outputs of a forward model [19]. As a polynomial evaluation is computationally fast, this approach becomes very useful when the original model has a high computational cost to be simulated. Then, some process where 
a lot of simulations are required, such as Sensitivity Analysis and Parameter fitting, become more practical to be performed.

Considering a vector $\boldsymbol{\xi}=\left(\xi_{1}, \xi_{2}, \ldots, \xi_{N}\right)^{T}$ of model inputs composed by independent random variables and assume that the quantity of interest $y$ is written in terms of these variables, it is possible to express this quantity through an infinite polynomial chaos expansion [12]. In practical applications, this quantity of interest can be approximated by a finite expansion obtained through a linear combination of the elements from the polynomial chaos basis:

$$
\bar{y}(\boldsymbol{\xi})=\sum_{i=1}^{P} b_{i} \Phi_{i}(\boldsymbol{\xi}),
$$

where $b_{i}$ are the unknown coefficients and $\Phi_{i}$ are orthogonal polynomial functions in terms of the inputs. This polynomial chaos expansion with $N$ inputs and order $d$ has $P$ terms, where $P=\frac{(N+d) !}{N ! d !}$.

Defined the approximation for the quantity of interest, as in Equation (3), it is necessary to determine the coefficients $b_{i}$ that define the polynomial in terms of the inputs. To this end, a non-intrusive approach named Probabilistic Collocation method is used, where a weighted residual formulation in the random space is defined [17] and the polynomial expansion needs to be equal to the model evaluation in a number of collocation points, which are samples of the random inputs. The result is a linear system in terms of the $b_{i}$ coefficients, then, the polynomial coefficients that approximate the quantity of interest are found by solving this system.

\subsection{Sensitivity Analysis}

In complex systems modeled as differential equations, like a Markov Chain with a large number of states and transitions between them, it is very common to appear a high number of parameters. In these cases, it is important to understand how each parameter influences the entire system.

A Sensitivity Analysis of the ten parameters associated in the $I_{C a L}$ current from the Pandit-Mahajan model was done in order to determinate which parameters have more influence on the main characteristics of the $I_{C a L}$ curve. To do this analysis, the ChaosPy library [6] implemented in Python was used.

The ChaosPy library has methods to quantify the uncertainty combining the use of the Monte Carlo method and also the PCE. To determine the PCE coefficients, it provides the Probabilistic Collocation method and the Pseudospectral Projection method.

The ten parameters, $\tau_{p o}, k_{1}, k_{2}, k_{1}^{\prime}, k_{2}^{\prime}, r_{1}, r_{2}, s_{1}, s_{2}$ and $g_{C a L}$, were analyzed and the results showed that three of them have more influence on the studied $I_{C a L}$ curve. Figure 2 shows these results. 


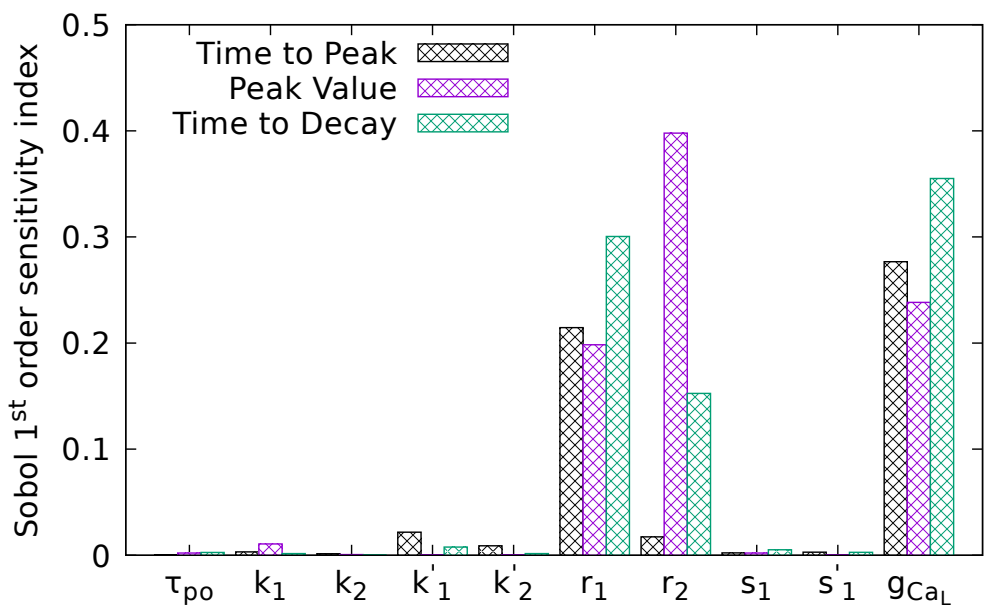

Fig. 2. The Sobol $1^{\text {st }}$ order sensitivity index ${ }^{4}$ of the parameters associated with the main $I_{C a L}$ curve characteristics: the Time to Peak, the Peak Value and the Time to Decay.

As can be seen in Figure 2, the parameters $r_{1}, r_{2}$ and $g_{C a L}$ have high influence on the main $I_{C a L}$ current from the Pandit-Mahajan model. So, considering these results, the search space of the Genetic Algorithm might be reduced from ten parameters to only three.

\subsection{Parameter Adjustment Using Genetic Algorithm}

A parameter adjustment using a Genetic Algorithm was used to adjust the LCC Markov Chain parameters so that the $I_{C a L}$ curve presented in Pandit original model remains unaltered in the new Pandit-Mahajan model. The GA was implemented using the "Simple Genetic Algorithm" provided by Pagmo C++ library [4].

Two important processes when defining a Genetic Algorithm are the choices of the variable of search (Genes) and the objective function (Fitness function). In order to evaluate the effectiveness of the Sensitivity Analysis done, the variables of the search were defined in two ways: the first with the ten parameters and, the second, with only the three parameters highlighted by the Sensitivity Analysis. The second process, the definition of the Fitness function, used the main characteristics of the $I_{C a L}$ curve. In this process, two methods were tested to evaluate the objective function: the first one was considering the Pandit-Mahajan mathematical model simulations and the second was considering the emulations done

\footnotetext{
${ }^{4}$ The $1^{\text {st }}$ order sensitivity index quantifies the portion that an input parameter contributes directly to the total variance of the quantity of interest, for more details see Eck et al.[5].
} 
by the PCE generated through the ChaosPy library. Mathematically, this process can be seen as a minimization problem with objective functions presented by the Equations (4) and (5) for the approach using the simulations of the model and the emulations using the $\mathrm{PCE}$, respectively.

$$
\begin{aligned}
F\left(v, I_{C a L_{p}}\right) & =\frac{1}{\left|\operatorname{TimeToPeak}\left(I_{C a L_{p}}\right)\right|}\left|\operatorname{TimeToPeak}(v)-\operatorname{TimeToPeak}\left(I_{C a L_{p}}\right)\right| \\
& +\frac{1}{\left|\operatorname{PeakValue}\left(I_{C a L_{p}}\right)\right|}\left|\operatorname{PeakValue}(v)-\operatorname{PeakValue}\left(I_{C a L_{p}}\right)\right| \\
& +\frac{1}{\left|\operatorname{TimeToDecay}\left(I_{C a L_{p}}\right)\right|}\left|\operatorname{TimeToDecay}(v)-\operatorname{TimeToDecay}\left(I_{C a L_{p}}\right)\right|
\end{aligned}
$$

where $v$ is the $I_{C a L}$ curve generated by the Pandit-Mahajan simulation to be evaluated by the function and $I_{C a L_{p}}$ is the original curve of the Pandit model.

$$
\begin{aligned}
F\left(\boldsymbol{\xi}, I_{C a L_{p}}\right) & =\frac{1}{\mid \text { TimeToPeak }\left(I_{C a L_{p}}\right) \mid} \mid P_{T T P}(\boldsymbol{\xi})-\text { TimeToPeak }\left(I_{C a L_{p}}\right) \mid \\
& +\frac{1}{\mid \text { PeakValue }\left(I_{C a L_{p}}\right) \mid}\left|P_{P V}(\boldsymbol{\xi})-\operatorname{PeakValue}\left(I_{C a L_{p}}\right)\right| \\
& +\frac{1}{\mid \text { TimeToDecay }\left(I_{C a L_{p}}\right) \mid} \mid P_{T T D}(\boldsymbol{\xi})-\text { TimeToDecay }\left(I_{C a L_{p}}\right) \mid
\end{aligned}
$$

where $\boldsymbol{\xi}$ are the set o parameters to be evaluated by the function, $P_{\bullet}(\boldsymbol{\xi})$ are the $4^{\text {th }}$ order PCE that approximate the "Time To Peak" $\left(\bullet_{T T P}\right)$, "Peak Value" $(\bullet P V)$ and the "Time To Decay" $\left(\bullet_{T T D}\right)$ features and $I_{C a L_{p}}$ is the original curve of the Pandit model.

The chromosome $C$ of the genetic algorithm is defined as $C=\left\{c_{p} \in \mathbb{R} \mid 0.1<\right.$ $\left.c_{p}<5.0\right\}$ where $c_{p}$ is the multiplier for the specific parameter $p$. All Genetic Algorithm executions were done with the standard setup from the Pagmo library except the use of the crossover strategy. In this study was used the Simulated Binary Crossover ("sbx") strategy, also provided by Pagmo library. All executions used 15 generations and the population was composed of 150 individuals.

\section{Results}

The main objective of this study is to evaluate a new process of parameter adjustment based on uncertainty quantification techniques and genetic algorithm.

After the use of the methods described in Section 2, two classes of results were generated. The first one using the simulations of the mathematical model into the GA evaluation and, the second one, using the emulations done by the Polynomial Chaos Expansions obtained using the ChaosPy library. Figure 3 shows the results obtained in the different tests. 


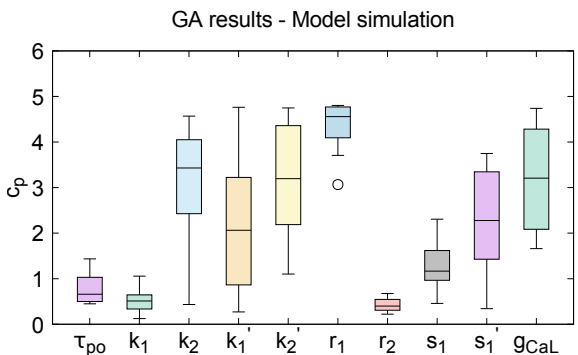

(a)

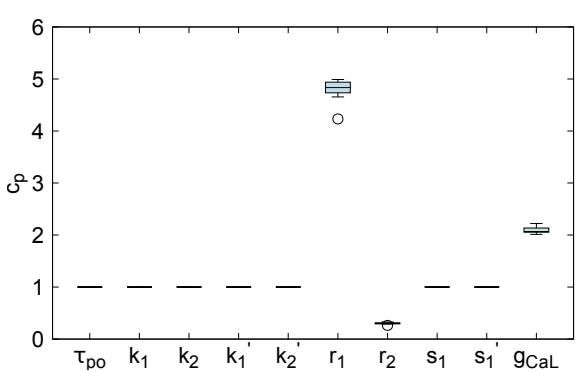

(c)

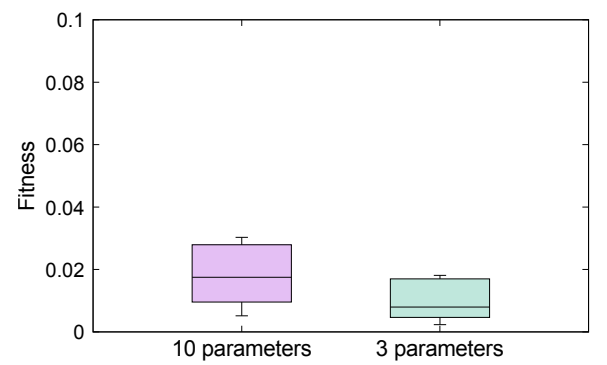

(e)

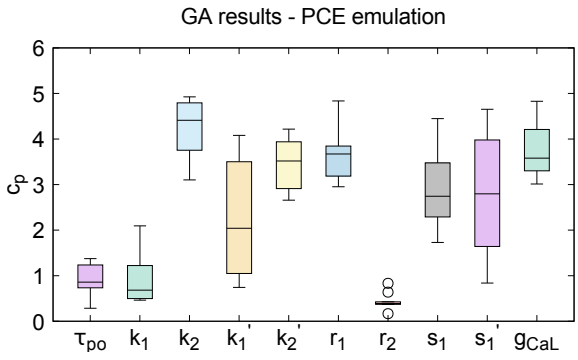

(b)

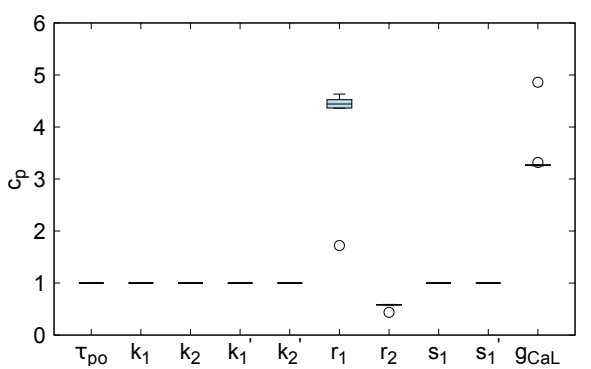

(d)

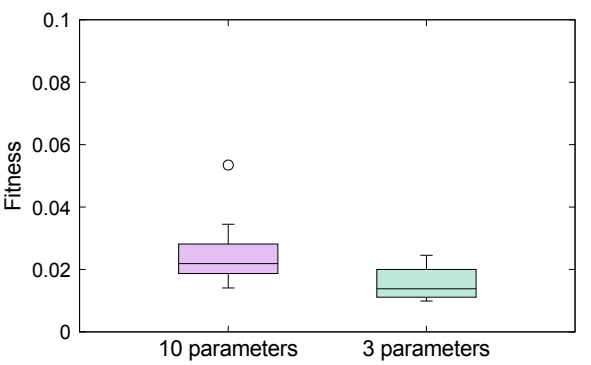

(f)

Fig. 3. Results obtained by the GA in the cases of study. The descriptive measures (minimum, first quartile, median, third quartile and maximum values) of the multiplier $c_{p}$ result adjusting 10 parameters using the mathematical model simulations (a) and the PCE emulations (b); the descriptive measures of the the multiplier $c_{p}$ result adjusting 3 parameters using the mathematical model simulations (c) and PCE emulations (d); the descriptive measures for the best Fitness found considering the simulations of the mathematical model (e) and emulations using the PCE (f) for 10 and 3 parameters.

The best results obtained by the GA using the simulation of Pandit-Mahajan model and the emulation by the PCE are presented in Tables 1 and 2, respectively. 
Table 1. The parameter values from the Original Model presented with the values encountered by the best execution of the Genetic Algorithm considering the mathematical model simulation using both the 10 parameters and the 3 parameters.

\begin{tabular}{cccc}
\hline Parameter & Mahajan et al. $[\mathbf{1 3}]$ & $\mathbf{1 0}$ parameters & 3 parameters \\
\hline$\tau_{p o}$ & 1 & $\mathbf{1 . 4 3 4}$ & 1 \\
$k_{1}$ & 0.03 & $\mathbf{0 . 0 1 5}$ & 0.03 \\
$k_{2}$ & $1.0410^{-4}$ & $\mathbf{3 . 8 8} \mathbf{1 0}^{-4}$ & $1.03610^{-4}$ \\
$k_{1}^{\prime}$ & $4.1310^{-3}$ & $\mathbf{4 . 3 6 1 \mathbf { 1 0 } ^ { - 3 }}$ & $4.1310^{-3}$ \\
$k_{2}^{\prime}$ & $2.2410^{-3}$ & $\mathbf{7 . 0 9 2} \mathbf{1 0}^{-3}$ & $2.2410^{-3}$ \\
$r_{1}$ & 0.3 & $\mathbf{1 . 2 4 7}$ & $\mathbf{1 . 4 8 2}$ \\
$r_{2}$ & 3 & $\mathbf{0 . 6 6 7}$ & $\mathbf{0 . 9 1 4}$ \\
$s_{1}$ & 0.02 & $\mathbf{0 . 0 2 3}$ & 0.02 \\
$s_{1}^{\prime}$ & $1.9510^{-3}$ & $\mathbf{6 . 6 7 1 ~ 1 0}$ & $1.9510^{-3}$ \\
$g_{C a L}$ & 0.031 & $\mathbf{0 . 0 5 2}$ & $\mathbf{0 . 0 6 4}$ \\
\hline
\end{tabular}

Table 2. The parameter values from the Original Model presented with the values encountered by the best execution of the Genetic Algorithm considering the mathematical model emulation done with the PCE using both the 10 parameters and the 3 parameters.

\begin{tabular}{cccc}
\hline Parameter & Mahajan et al. . $[\mathbf{1 3}]$ & $\mathbf{1 0}$ parameters & 3 parameters \\
\hline$\tau_{p o}$ & 1 & $\mathbf{1 . 2 3 2}$ & 1 \\
$k_{1}$ & 0.03 & $\mathbf{0 . 0 2 3}$ & 0.03 \\
$k_{2}$ & $1.03610^{-4}$ & $\mathbf{4 . 0 9} \mathbf{1 0}^{-4}$ & $1.03610^{-4}$ \\
$k_{1}^{\prime}$ & $4.1310^{-3}$ & $\mathbf{0 . 0 1 4}$ & $4.1310^{-3}$ \\
$k_{2}^{\prime}$ & $2.2410^{-3}$ & $\mathbf{9 . 4 4 8} \mathbf{1 0}$ & $2.2410^{-3}$ \\
$r_{1}$ & 0.3 & $\mathbf{0 . 9 8 8}$ & $\mathbf{1 . 3 0 9}$ \\
$r_{2}$ & 3 & $\mathbf{1 . 2 7 9}$ & $\mathbf{1 . 7 3 1}$ \\
$s_{1}$ & 0.02 & $\mathbf{0 . 0 4 3}$ & 0.02 \\
$s_{1}^{\prime}$ & $1.9510^{-3}$ & $\mathbf{7 . 9 3 9} \mathbf{1 0}$ & $1.9510^{-3}$ \\
$g_{C a L}$ & 0.031 & $\mathbf{0 . 1 1 5}$ & $\mathbf{0 . 1 0 1}$ \\
\hline
\end{tabular}

Figure 4 shows the $I_{C a L}(4(\mathrm{a}))$, AP $(4(\mathrm{~b}))$ and $[C a]_{i}(4(\mathrm{c}))$ curves of the proposed Pandit-Mahajan model after the parameter adjustment using the 3 parameter results considering both simulations of the model (PM-SIM) and emulation of the PCE (PM-EMU) in comparison with the original Pandit model. 


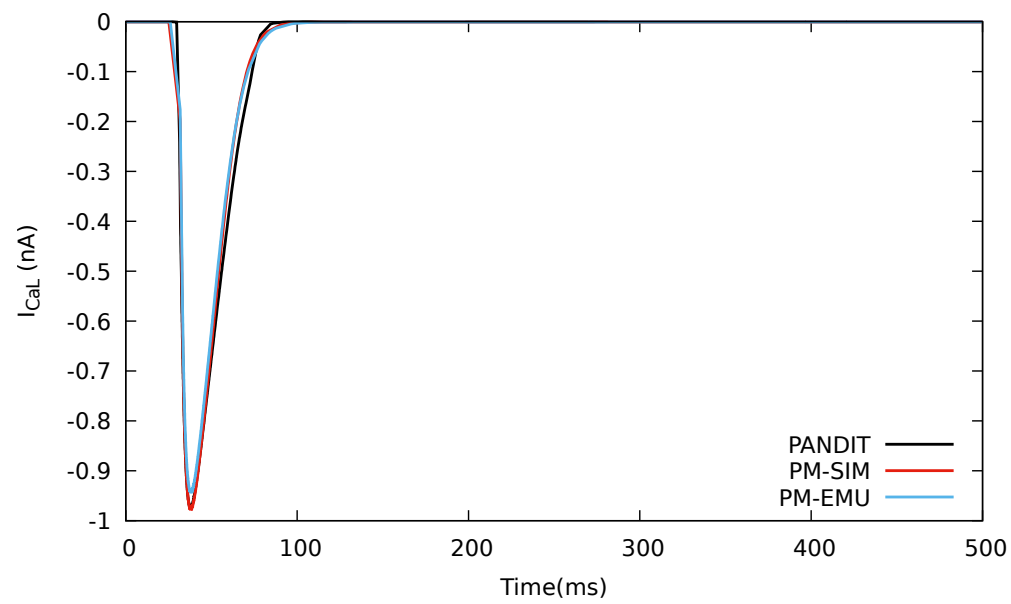

(a)

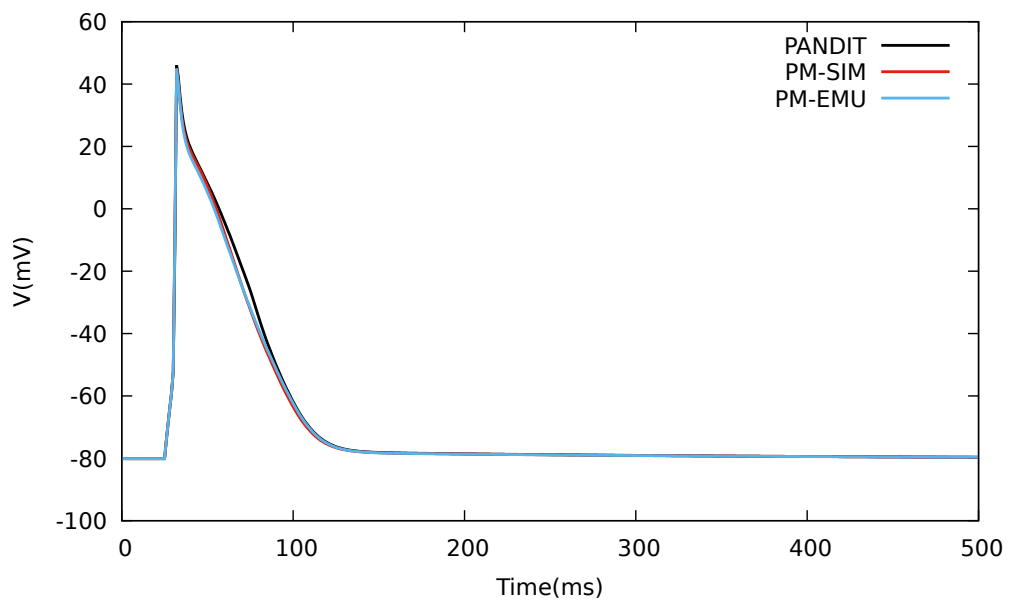

(b)

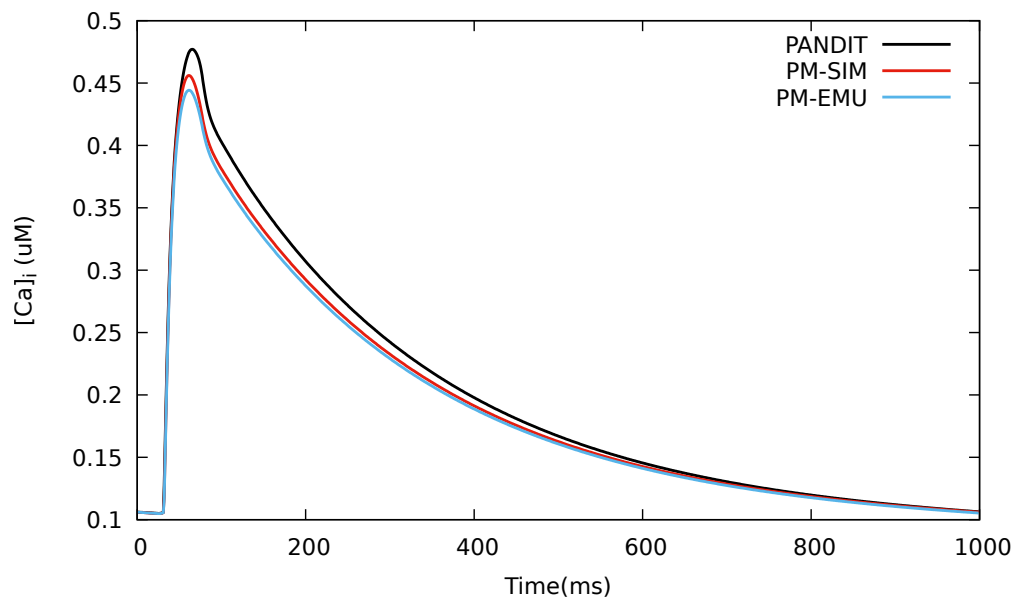

(c)

Fig. 4. $I_{C a L}$ current (a), Action Potential (b) and $[\mathrm{Ca}]_{i}$ (c) curves generated by Pandit original model (PANDIT) compared with the new Pandit-Mahajan model after the best parameter adjustment using the simulation of the model (PM-SIM) or the emulation of the PCE (PM-EMU) into the Genetic Algorithm search process. 
As can be seen, the GA adjustment was able to fit the new model formulations in order to maintain the main characteristics of the $I_{C a L}$ curve as presented in the original model using both simulation and emulation techniques.

Since the $I_{C a L}$ curve was well reproduced, the main electrophysiology variables, the Action Potential (AP) and the Intracellular Calcium Concentration $\left([\mathrm{Ca}]_{i}\right)$, also were well reproduced.

\subsection{Dimensional Reduction}

The first method studied was the Dimensional Reduction. Based on the Sensitivity Analysis done, it was possible to notice that three parameters have more influence on the main features of the $I_{C a L}$ curve.

The GA was executed 10 times with the search space of 10 parameters and 10 times with search space of 3 parameters. The values found by the GA in both cases, using the simulation of the model or the emulation by the PCE, are shown in the Figures 3(a), 3(c) and in Figures 3(b) and 3(d), respectively.

As can be seen in Figures 3(e) and 3(f), the reduction in the search space dimension of the GA generated an increase in the quality of the results obtained.

\subsection{Emulations}

An Emulator based on PCE generated by ChaosPy library was tested instead of the mathematical model in the GA Fitness evaluation. Figures 3(b) and 3(d) show the results obtained by the GA using the Emulation technique.

When compared with the GA results using the mathematical model (Figures 3(b) and 3(d)), the use of the Emulation obtained satisfactory results. The values found by GA were similar using both simulations of the mathematical model or using emulations. Furthermore, even changing the evaluation method, the obtained Fitness values were similar using both methods as can be seen in Figures $3(\mathrm{e})$ and $3(\mathrm{f})$.

The great advantage of using the Emulation technique is associated with the computational cost. The same results considering both techniques was obtained by the GA but the search process using the simulations as evaluation function took approximately 90 minutes to find the results, whereas using the emulation it took less than 1 second. Considering that ChaosPy took around of 30 minutes to generate the PCE, it is possible to conclude that the use of the Emulation technique speeds up the problem solution at least in 3 fold.

\section{Conclusions and Discussions}

The coupling of different models or even an update of an existing model is an important tool to develop new electrophysiological studies. However, this process involves different procedures and techniques that imply a set of parameter adjustments, which may determinate the quality of the results. 
In this paper, we presented a new method based on the combination of Polynomial Chaos expansion and a Genetic Algorithm to optimize the efficiency and the efficacy of the process of coupling two different models. Furthermore, a dimensional reduction in the Genetic Algorithm search space was done based on the Sensitivity Analysis, which showed that for the Pandit-Mahajan specific coupling process, only three parameters have the biggest influence in the parameter estimations needed to model $I_{C a L}$ curve. This result motivated an adjustment involving only these three parameters instead of the ten previously adjusted. This reduction showed to be a good option since it generated smaller Fitness values when adjusted with the Genetic Algorithm. This reduction may be explained due to the fact that this dimensional reduction in the Genetic Algorithm search space, considering now only the more important parameters, facilitated the process to find the optimal values.

Another important point to highlight is that, considering all the ten parameters, the Genetic Algorithm results did not converge to a small range of values. The results of the parameters multipliers $c_{p}$ assumed different values in each algorithm execution. This behavior is not interesting since the results are associated with parameters that, in some cases, are related to the biological characteristics of the model. So this variation may cause a decrease in confidence of its value. This unwanted behavior did not occur in the Genetic Algorithm considering the Sensitivity Analysis. With the combined techniques, the results had converged to a small range of values and, with this behavior, they may be more trustworthy.

The Fitness evaluation process in a Genetic Algorithm can be a computationally expensive process, mainly when this process involves a simulation of a mathematical model. In this paper, a method using an Emulator based in Polynomial Chaos Expansions was tested instead of the mathematical model in order to replace a complex model simulation for a single polynomial evaluation and, with this, reduce the cost associated with this process.

The Polynomial Chaos Expansions method was more effective than the Genetic Algorithm since it was able to obtain the roughly the same results, however, reducing the Genetic Algorithm search process from 90 minutes to less than 1 second in the configuration used in this paper. If we consider a larger number of generations or a bigger population size of the Genetic Algorithm, the speed up will be highly increased.

In conclusion, in this paper, we employ a parameter adjustment process combining Polynomial Chaos Expansions and Genetic Algorithm to couple two models: Pandit and Mahajan into a new combined Pandit-Mahajan model. The procedure showed to be effective to facilitate the coupling of the two models since it arrives in excellent agreement with the original model.

\section{References}

1. Armstrong, C.M., Bezanilla, F.: Inactivation of the sodium channel. ii. gating current experiments. The Journal of general physiology 70(5), 567-590 (1977) 
2. Beeler, G.W., Reuter, H.: Reconstruction of the action potential of ventricular myocardial fibres. The Journal of physiology 268(1), 177-210 (1977)

3. Bezanilla, F., Armstrong, C.M.: Inactivation of the sodium channel. i. sodium current experiments. The Journal of general physiology 70(5), 549 (1977)

4. Biscani, F., Izzo, D.: esa/pagmo2: pagmo 2.9 (Aug 2018)

5. Eck, V.G., Donders, W.P., Sturdy, J., Feinberg, J., Delhaas, T., Hellevik, L.R., Huberts, W.: A guide to uncertainty quantification and sensitivity analysis for cardiovascular applications. International journal for numerical methods in biomedical engineering 32(8), e02755 (2016)

6. Feinberg, J., Langtangen, H.P.: Chaospy: An open source tool for designing methods of uncertainty quantification. Journal of Computational Science 11, 46-57 (2015)

7. Gattoni, S., Røe, Å.T., Frisk, M., Louch, W.E., Niederer, S.A., Smith, N.P.: The calcium-frequency response in the rat ventricular myocyte: an experimental and modelling study. The Journal of physiology 594(15), 4193-4224 (2016)

8. Hauseux, P., Hale, J.S., Cotin, S., Bordas, S.P.: Quantifying the uncertainty in a hyperelastic soft tissue model with stochastic parameters. Applied Mathematical Modelling (2018)

9. Hodgkin, A.L., Huxley, A.F.: A quantitative description of membrane current and its application to conduction and excitation in nerve. The Journal of physiology 117(4), 500-544 (1952)

10. Lawson, B.A., Burrage, K., Burrage, P., Drovandi, C.C., Bueno-Orovio, A.: Slow recovery of excitability increases ventricular fibrillation risk as identified by emulation. Frontiers in physiology 9 (2018)

11. Lawson, B.A., Drovandi, C.C., Cusimano, N., Burrage, P., Rodriguez, B., Burrage, K.: Unlocking data sets by calibrating populations of models to data density: A study in atrial electrophysiology. Science advances 4(1), e1701676 (2018)

12. Li, H., Zhang, D.: Probabilistic collocation method for flow in porous media: Comparisons with other stochastic methods. Water Resources Research 43(9) (2007)

13. Mahajan, A., Shiferaw, Y., Sato, D., Baher, A., Olcese, R., Xie, L.H., Yang, M.J., Chen, P.S., Restrepo, J.G., Karma, A., et al.: A rabbit ventricular action potential model replicating cardiac dynamics at rapid heart rates. Biophysical journal 94(2), 392-410 (2008)

14. Pandit, S.V., Clark, R.B., Giles, W.R., Demir, S.S.: A mathematical model of action potential heterogeneity in adult rat left ventricular myocytes. Biophysical journal 81(6), 3029-3051 (2001)

15. Ratto, M., Castelletti, A., Pagano, A.: Emulation techniques for the reduction and sensitivity analysis of complex environmental models (2012)

16. Stary, T.: Mathematical and computational study of markovian models of ion channels in cardiac excitation (2016)

17. Tatang, M.A., Pan, W., Prinn, R.G., McRae, G.J.: An efficient method for parametric uncertainty analysis of numerical geophysical models. Journal of Geophysical Research: Atmospheres 102(D18), 21925-21932 (1997)

18. Terkildsen, J.R., Niederer, S., Crampin, E.J., Hunter, P., Smith, N.P.: Using physiome standards to couple cellular functions for rat cardiac excitation-contraction. Experimental physiology 93(7), 919-929 (2008)

19. Xiu, D.: Numerical methods for stochastic computations: a spectral method approach. Princeton university press (2010)

20. Yanagihara, K., Noma, A., IRISAWA, H.: Reconstruction of sino-atrial node pacemaker potential based on the voltage clamp experiments. The Japanese journal of physiology 30(6), 841-857 (1980) 